\title{
La situación cambiante de la universidad en la era digital
}

\section{The Changing Situation of University in the Digital Age}

\author{
Juana M. Sancho Gil \\ Universidad de Barcelona (España)
}

Adriana Ornellas

Universitat Oberta de Catalunya (España)

Judith Arrazola Carballo

Universidad de Barcelona (España)

\section{Resumen}

En los últimos cuarenta años se han producido extensos y profundos cambios políticos, económicos, sociales y tecnológicos, mediados por los movimientos neoconservadores y el aparentemente imparable desarrollo de la tecnología digital. Aunque, al mismo tiempo, temas fundamentales como las relaciones asimétricas de poder, la desigualdad, la pobreza y la exclusión no sólo han permanecido inalterables, sino que se están acentuando, produciendo, en ciertos aspectos, movimientos y tendencias de cambio involutivo. Desde esta perspectiva, en este artículo, comenzamos dialogando sobre el sentido de los cambios en la universidad, a partir de las aportaciones de distintos autores interesados en el tema. Seguimos situando la problemática considerando los efectos de las transformaciones producidas por las tecnologías digitales en la vida y la experiencia profesional de los docentes e investigadores universitarios. La investigación llevada a cabo nos permite situar el impacto de la proliferación sin precedentes de la información y el uso de aplicaciones digitales en la noción de conocimiento y en los métodos de enseñanza y aprendizaje, así como vislumbrar los desafíos que se le presentan a la universidad. El texto finaliza con la consideración de algunos de los retos y perspectivas universitarias actuales, a partir de distintos estudios y propuestas internacionales. Nuestras conclusiones invitan al lector a reconsiderar necesariamente el sentido y el papel de la tecnología digital, no solo en la transformación aparentemente inevitable de la universidad, sino también en el de la sociedad.

Palabras clave: cambio tecnológico; innovación; educación superior; cambio social; condiciones de trabajo.

\section{Abstract}

Extensive and profound political, economic, social and technological changes have taken place over the last forty years, mediated by neoconservative movements and the apparently 
unstoppable development of digital technology. At the same time, however, fundamental issues such as asymmetric power relations, inequality, poverty and exclusion have not only remained unchanged but are becoming more pronounced, producing, in some respects, movements and trends of reactionary change. From this perspective, in this article we begin by discussing the meaning of changes in the university field, based on contributions from different authors interested in the subject. We continue to analyze the problem considering the effects of the transformations produced by digital technologies on the lives and professional experience of university teachers and researchers. The evidence provided by this research project allows us to situate the impact of the unprecedented proliferation of information and the use of digital resources on the notion of knowledge and on teaching and learning methods, as well as to envisage the challenges to face by university. The text concludes considering some of the current university challenges and perspectives, based on various international studies and proposals. Our conclusions invite the reader to necessarily question the sense and the role of digital technology, not only in the seemingly inevitable transformation of university, but also in that of society.

Keywords: technological change; innovation; higher education; social change; working conditions.

Everything changes, nothing remains without change.

(Siddhārtha Gautama)

Como nos recuerda Siddhārtha Gautama (Buda), todo cambia, nada permanece sin cambio. Un principio que, en la década de 1990, llevó a autores como Peter Senge, a argumentar que, en el nuevo contexto político, económico, social y tecnológico, sólo sobrevivirían las instituciones “capaces de aprender”. Mediante el análisis de los procesos institucionales, este autor se proponía "destruir la ilusión de que el mundo está creado por fuerzas separadas y no relacionadas”. Para él, si se lograba dejar de lado esta ilusión, sería posible crear “organizaciones que aprenden”. Es decir, organizaciones en las que los individuos amplían de manera continua su capacidad para crear los resultados que realmente desean, en las que se fomentan formas nuevas y abiertas de pensar, en las que las aspiraciones colectivas se expresan libremente, y en las que la gente aprende continuamente cómo aprender conjuntamente.

Desde su perspectiva, "las organizaciones que realmente sobresaldrán en el futuro serán las que descubran cómo conseguir el compromiso y la capacidad de aprender de todos los niveles de una organización” (Senge, 1990, pp. 3-4).

Casi treinta años después, los nuevos contextos políticos, económicos, sociales y tecnológicos, mediados por los movimientos neoconservadores y al aparentemente imparable desarrollo de la tecnología digital, han propulsado cambios sin precedentes. Aunque, al mismo tiempo, temas fundamentales como las relaciones asimétricas de poder, la desigualdad, la pobreza y la exclusión no sólo permanecen inalterables, sino que se acentúan, produciendo en ciertos aspectos un cambio involutivo. Con lo que, como argumentaba, hace algún tiempo Pareto (1966), es ese contraste entre lo 
que cambia y lo que persiste, o en algunos casos empeora, lo que provoca las mayores turbulencias.

Desde esta perspectiva, en este artículo comenzamos problematizando el sentido del cambio en la universidad. Seguimos con algunas evidencias proporcionadas por nuestra experiencia e investigación sobre la influencia de la digitalización de la sociedad en el trabajo del profesorado universitario. El texto finaliza con el análisis de algunos retos y perspectivas que se vislumbran para el futuro.

\section{LAS MÚLTIPLES DIMENSIONES DEL CAMBIO EN LA UNIVERSIDAD}

La tendencia de denominar las diferentes etapas de evolución de la humanidad por la tecnología o las formas de organización y producción dominantes (edad de bronce, de hierro, sociedad industrial, postindustrial, del conocimiento, de la información de la red y digital), puede contribuir a minimizar el contexto y el sentido de las fuerzas ideológicas que informan su desarrollo. De ahí que en este monográfico sobre la "universidad digital", en este artículo no nos centraremos en el impacto de las tecnologías digitales en las organizaciones de educación superior. Como hemos apuntado, nuestra mirada se centra en las realidades sociales, culturales y económicas que configuran el momento actual, sus efectos en el profesorado y los retos que se vislumbran para la universidad.

En la llamada sociedad digital, las corrientes neoconservadoras no son ajenas al desarrollo de la tecnología que le da nombre. Y no lo son, en primer lugar, porque en las decisiones sobre el sentido de sus desarrollos priman, una vez más, las visiones economicistas y consumistas. Lo que llevó a Andrew Russell a afirmar:

Every jump in technology draws attention and capital away from existing technologies used by the 99 percent, which therefore undermines equality, and reduces the ability for people to get onto the 'playing field' in the first place [...] I have never been able to understand why anyone would assume that the future would be evenly distributed [...] To put this in a different way: I have never been able to find any evidence from human history to suggest that the future will be evenly distributed (LaFrance, 2016, $\mathrm{s} / \mathrm{p})$.

Por lo que, como argumentan Chatterton y Newmarch (2017), desde el propio título de su artículo, "the future is already here-it's just not very evenly distributed".

En segundo lugar, porque en su uso se priorizan modelos de negocio orientados, en general, a conseguir ganancias millonarias, sin necesariamente pensar en la redistribución social a través de un pago justo de impuestos (Kenney, 2017) y en la cantidad y calidad del empleo generado (Stone, 2017). En tercer lugar, porque la producción y difusión masivas de información que propician, además de no significar de forma automática más conocimiento, tienden a aumentar los niveles de fraude y 
engaño y el uso indiscriminado de la información proporcionada -de forma más o menos consciente, por los usuarios de los distintos servicios proporcionados a través de la Web (Sancho y Hernández, 2018). En cuarto lugar, porque en un momento de auge de los movimientos populistas más variados (Akbaba y Jeffrey, 2017) contribuyen de forma significativa a magnificar los efectos de llamada posverdad, actuando de eco de las circunstancias en las que los hechos objetivos influyen menos en la formación de la opinión pública que los llamamientos a la emoción y la creencia personal. Y, en quinto lugar, porque los nuevos sistemas de información están alimentando las visiones de la educación más como negocio (Ametller, 2016; Knapp y Siegel, 2009) que como bien social (UNESCO, 2015a).

En este sentido cabe destacar que, en el caso de España, como sucede en otros muchos países, mientras que las universidades públicas se han visto afectadas por considerables recortes achacados a la "crisis" (por ejemplo, en algunas facultades de la Universidad de Barcelona, el $72 \%$ del profesorado tiene contratos temporales y precarios), las 14 universidades creadas en lo que va de siglo son privadas, y cuatro de ellas no presenciales. Esta situación nos ha de llevar a reflexionar sobre las posibles consecuencias que esta tendencia puede tener en la articulación de la sociedad y en el aumento de la brecha económica y social (ITPI, 2016; Martínez, 2013; Rizvi, 2016; Shavit, Arum, Gamoran y Menahem, 2007).

Por otro lado, en relación al fenómeno de creciente desigualdad económica y social, como sugieren Miñana y Rodríguez (2003), también resulta necesario pensar sobre el sentido de la insistencia en el valor de las 'innovaciones', las pruebas de evaluación de competencias, los estándares, la comparación de resultados, (y la utilización acrítica, en algunos casos, de la tecnología digital, añadiríamos nosotras), como parte del discurso de la calidad y la eficacia. Todo ello con la finalidad de mostrar diferencias que lleven a los consumidores a elegir las opciones más ventajosas.

Para Galcerán (2010, p.14), este panorama no resulta novedoso ya que "la universidad ha sido siempre una institución productora de cultura ligada al poder y, en las sociedades capitalistas, una institución de reproducción social de ese tipo de sistema". Aunque quizás hoy resulte más evidente cómo "el potencial de creación de saber, conocimiento y cultura de las universidades está siendo atrapado por los intereses empresariales" (p. 15). Este movimiento no es casual, sino que viene impulsado por el denominado capitalismo cognitivo (Moulier Boutang, 2012; Rindermann, 2018) y por la lucha por atraer al máximo número de estudiantes, a quienes se les "anima a que conciban su formación como un «capital cultural» del que podrán disponer en un trabajo futuro, algo así como una inversión: el joven invierte en su formación y esa inversión le dará réditos en un futuro" (Galcerán, 2010, p. 17). Para esta autora, todo ello propicia el crecimiento de la noción de universidad-empresa, por "su incorporación a los circuitos empresariales y mercantiles de la sociedad capitalista actual" (p. 15) y por la tendencia a trasladar la estructura gerencial de la empresa a la universidad, "haciendo recaer los cargos de dirección en personal externo, al estilo de un gerente de empresa, reduciendo 
los órganos colegiados y estableciendo criterios de rentabilidad para la concesión de plazas y financiación” (p. 19), siendo, a menudo, el cálculo económico el único criterio a tener en cuenta. Aunque "desafortunadamente, para muchos estudiantes y jóvenes intelectuales el futuro que la universidad-empresa prepara para ellos no es nada apetecible (p. 34).

Para esta autora, las transformaciones actuales de la universidad tienen un gran alcance debido a la puesta en marcha del mercado global de la educación superior y a la primacía de una noción de investigación que, al igual que la enseñanza, se enfoca claramente hacia los intereses del mercado. Todo ello en detrimento de amplios ámbitos, sobre todo, de las Humanidades y las Ciencias Sociales.

En el tema particular de las Tecnologías de la Información y la Comunicación (TIC), herramientas fundamentales de la universidad del siglo XXI, autores como Vercellone (2010) argumentan la necesidad de señalar que la transformación de la Universidad no puede ser explicada por el "determinismo tecnológico que hace de las TIC (Tecnologías de la Información y la Comunicación) el principal factor en el paso a una nueva organización del trabajo y de las relaciones sociales" (p. 84). Dado que su desarrollo y uso implican la disposición, la toma de decisiones y el saber de un conjunto de seres humanos con formas determinadas de situarse en el mundo y posicionarse ante el tipo de sociedad que están dispuestos a contribuir a crear. Aunque la tecnología digital es considerada como condición previa e ineludible para participar en los beneficios del nuevo proyecto social: la sociedad de la información o del conocimiento (Perdomo Reyes, 2006). En este sentido, resulta imprescindible no olvidar que una de las características fundamentales de estas tecnologías

es justamente que el lenguaje y su capacidad interactiva son propiedades intrínsecas. En los segmentos o en las aplicaciones postfordistas ya no basta con la ejecución de complejos protocolos, ni con la rutina del gesto o del movimiento repetido una y mil veces. Se trata de exigir a la ciudadanía, una parte de la cual ha sido convertida en neoproletariado polivalente, que se implique, sea sumisa, maleable, dentro y fuera del trabajo y de este modo conseguir la excelencia en competitividad (Ferreiro, 2010, p. 116).

Todo ello conlleva importantes consecuencias para la vida y el trabajo de los docentes universitarios.

\section{DE CÓMO LO DIGITAL AFECTA A LOS DOCENTES UNIVERSITARIOS}

El desarrollo de las tecnologías digitales no sólo ha propiciado nuevas formas de entender la universidad, sino que está teniendo importantes consecuencias en quienes trabajan en ella, en particular, en los docentes e investigadores. Lo que resulta más evidente son los cambios en la forma de entender la docencia en las instituciones 
no presenciales. Tanto en las que comenzaron "sobre papel", que han incorporado con presteza los campus virtuales y todas sus tecnologías complementarias, como las ya mediadas, desde su fundación, por la tecnología digital. En estos casos, queda claro que el personal al cargo de la docencia, además de los conocimientos y las habilidades tradicionales, ha tenido que desarrollar o adquirir nuevas competencias y saberes relacionadas con las posibilidades y limitaciones de estas tecnologías y el tipo de relación docente que propician (Anderson, 2004). Pero los docentes de las universidades presenciales también reconocen sentirse afectados por este nuevo escenario tecnológico y cultural. A ese respecto, diversos autores han realizado propuestas sobre los roles y competencias de los docentes en situaciones de enseñanza y aprendizaje en línea. Aquí cabe destacar el estándar de competencia en TIC para docentes de la UNESCO (2011), que contempla seis dimensiones: a) la comprensión de la función de las TIC en la educación; b) los planes de estudio y la evaluación; c) la pedagogía; d) las TIC; e) la organización y la gestión y f) la formación profesional de los docentes.

En este contexto, como parte del proyecto de I+D+I: Los efectos de los cambios sociales en la vida profesional de los docentes universitarios, entre 2007 y 2010, elaboramos 24 historias de vida profesional con otros tantos docentes e investigadores universitarios con más de 20 años de servicio y pertenecientes a universidades presenciales históricas y de nueva creación (Sancho, 2013a). Nos encontrábamos en el inicio de una importante reestructuración en términos de gestión económica, nuevas formas de control y rendimiento de cuentas, prácticas de privatización, etc. Además de estar inmersos en pleno proceso de construcción de Espacio Europeo de Educación Superior. Uno de los cambios más significativos estaba relacionado con la considerable expansión de los estudios universitarios, que había conllevado a pasar de las 12 universidades históricas existentes hasta 1960, a las 50 públicas y las 25 privadas en el momento que realizamos este estudio. Número que ha seguido creciendo hasta hoy, con 51 públicas, una de ellas no presencial y 33 privadas, 4 no presenciales. De ahí que el principal objetivo de la investigación fuera profundizar nuestra comprensión sobre el impacto del cambio económico, social, cultural, tecnológico y laboral que estaban experimentando las universidades españolas en la vida y la identidad profesional del personal docente e investigador, teniendo en cuenta el contexto nacional y europeo.

En la investigación participaron 24 docentes e investigadores de distintas áreas de conocimiento, 12 hombres y 12 mujeres, 14 de ellos de 7 universidades históricas y 10 de universidades de nueva creación de 8 comunidades autónomas (tabla 1). 
Tabla 1. Características de la muestra intencional

\begin{tabular}{|l|l|l|l|}
\hline \multicolumn{1}{|c|}{ Universidad } & \multicolumn{1}{c|}{ Región } & \multicolumn{1}{c|}{ Género } & \multicolumn{1}{c|}{ Especialización } \\
\hline Histórica & CA1 & Hombre & Filosofía \\
\hline Histórica & CA1 & Mujer & Matemáticas \\
\hline Nueva creación & CA1 & Hombre & Filología gallega \\
\hline Histórica & CA2 & Mujer & Farmacia \\
\hline Histórica & CA2 & Hombre & Educación \\
\hline Nueva creación & CA2 & Hombre & Economía \\
\hline Nueva creación & CA3 & Hombre & Medicina Forense \\
\hline Nueva creación & CA3 & Mujer & Educación Musical \\
\hline Histórica & CA4 & Mujer & Biología Celular \\
\hline Histórica & CA4 & Hombre & Ciencias Políticas \\
\hline Nueva creación & CA4 & Hombre & Química y agricultura \\
\hline Nueva creación & CA4 & Mujer & Ciencias Sociales \\
\hline Histórica & CA5 & Mujer & Ciencias Sociales \\
\hline Histórica & CA5 & Mujer & Química \\
\hline Nueva creación & CA5 & Hombre & Ciencia y tecnología \\
\hline Histórica & CA6 & Mujer & Farmacia \\
\hline Histórica & CA6 & Hombre & Francés \\
\hline Nueva creación & CA6 & Mujer & Paleontología \\
\hline Histórica & CA7 & Hombre & Física cósmica \\
\hline Histórica & CA7 & Mujer & Derecho \\
\hline Nueva creación & CA7 & Mujer & Ingeniería \\
\hline Histórica & CA8 & Hombre & Informática \\
\hline Histórica & CA8 & Mujer & Bellas Artes \\
\hline Nueva creación & CA8 & Hombre & Ingeniería Industrial \\
\hline & & & \\
\hline
\end{tabular}

La elaboración de las historias de vida profesional (Goodson y Hargreaves, 1996) conllevó el análisis de distintos documentos sobre las condiciones laborales de las universidades implicadas y la realización de dos entrevistas en profundidad en los lugares de trabajo de los participantes, completadas con observaciones de estos mismos entornos. Además de un conjunto de intercambios y conversaciones a distancia que comenzaron con el proceso de negociación y acabaron con la aprobación final de los textos que se harían públicos por parte de los colaboradores.

Desde las vivencias y experiencias de los participantes en la investigación sobre los cambios experimentados en la universidad, en relación a la tecnología digital, destacaban: (i) las nuevas visiones sobre el conocimiento, (ii) la posibilidad de acceder a un sin número de fuentes de información, (iii) mayores facilidades para participar en proyectos internacionales y visibilizar el trabajo, y, sobre todo, (iv) el 
cambio en las expectativas y experiencias y predisposiciones del alumnado, que ya no consideraba ni a la universidad ni al profesorado como fuentes únicas e indiscutibles de saber.

En relación a las transformaciones sobre la noción de conocimiento, como explicaba una profesora,

en el ámbito en el que yo trabajo, los cambios han sido espectaculares. Porque el área de Matemáticas Aplicadas, en el año 82 no se sabía bien lo que era. Era una cosa que estaba surgiendo [...] se consideraba que... bueno, es sólo meter las cosas en el ordenador y tal... [...] Pero han ido pasando los años, y cada vez más las empresas para el desarrollo y la innovación de nuevas tecnologías, ven que la Matemática Aplicada puede aportar mucho conocimiento. Con lo cual hemos llegado a un momento en que, de no tener casi problemas reales encima de la mesa, hemos pasado a estar saturados (Sancho, 2013b, p. 116).

$\mathrm{O}$, como apuntaba un profesor de ingeniería, a las maneras de acceder al conocimiento disponible,

Profundizando en lo que supone la Internet, lo que sí noto ahora es much a diferencia en lo que supone la búsqueda de información, de documentación. Antes era "dogma" el limitarse a la información accesible a través de las revistas que pudiera tener tu universidad o alcanzable a través de colegas muy recelosos de compartirla y ahora la dificultad de cualquier persona es cómo escudriñar en el mundo de la información disponible a través de los múltiples bancos de información a los que las universidades están suscritas, o de libre disposición en la red... Cualquier buscador de artículos científicos te da acceso a un mundo, lo difícil es escudriñarlo. El problema que se tiene ahora, de limitar la información, no existía antes. Tener 15 o 20 buenos artículos para tu tesis era disponer de un tesoro a preservar (Romero y Rifà, 2013, p. 257).

Por otra parte, el ordenador e internet se estaban convirtiendo en unas herramientas imprescindibles que facilitaban e intensificaban a la vez el trabajo académico.

Todo se hace ahora vía Internet: evaluación de proyectos, evaluación de expedientes, evaluar publicaciones para revistas científicas, el acceso a bases de datos científicas... Esto unido a la cantidad de informes, proyectos y demás documentos que tienes que preparar periódicamente hace que la mayor parte de tu tiempo de trabajo transcurra frente al ordenador. Esto ha sido un gran cambio, el mayor que he experimentado en mi vida universitaria: pasar del laboratorio al ordenador, con sus ventajas e inconvenientes, pero con un balance muy positivo. El correo electrónico ha permitido mejorar las relaciones con otros grupos, así como los rendimientos de esos contactos. El poder estar en contacto permanente, intercambiar opiniones e informaciones de manera rápida, ha supuesto una mejora 
en el establecimiento de redes y grupos de trabajo (Creus y Gordaliza, 2013, pp. 329 - 330).

En este nuevo panorama, que comporta "una cantidad de ventajas y una cantidad de información que a veces hay que seleccionar porque abruma" (Rubio, Sancho y Costas, 2013, p. 98), junto con la posibilidad de utilizar nuevos recursos, comenzaba a conllevar cambios significativos en la concepción de los roles docentes y discentes, los modos de enseñar y aprender y el valor y sentido de la información. Con todas las posibilidades y limitaciones que suelen conllevar los cambios, tal como señalaba un profesor de Lingüística,

en una palabra, mi labor es orientar. Eso me exige un trabajo a mayores que es tratar de digerir, porque estar informado de toda la cantidad inmensa de información que nos está llegando... Antes era más cómodo y ahora es más divertido. Antes era más cómodo en el sentido de que tienes tus libros en tu biblioteca, tu bibliografía básica, tus ejercicios, tu librito de no sé qué, tu manualito y tu pizarra. [...] De repente, [...] todos estos avances llegan al aula, poner el ordenador, proyectar [...]-“Esto os lo mando a vuestro correo electrónico, puedes cogerlo de esta página”. [...] - "Podéis acceder a esta página que tiene mucha información, cuidado con ésta que esta información es buena pero ésta no tanto o ésta está un poco tergiversada"..., [...] no domino todas las tecnologías, pero hay gente que domina el trabajo con vídeos, el trabajo con subtitulación, etc. (Ibídem, p. 98).

\section{Y uno de Economía.}

A lo largo de mi carrera académica he ido variando los métodos de enseñanza y los materiales que utilizo para impartir las clases. En la actualidad utilizo una plataforma en la que "deposito" los recursos que necesitan los alumnos para estudiar las diferentes materias, concretamente resúmenes de todos los temas a los que tienen acceso los alumnos [...] Antes impartía clases magistrales en las que se exponía el marco teórico y los alumnos tomaban apuntes. Ahora me preparo las clases utilizando el Power Point, transparencias... Intento hacer las presentaciones más ilustrativas y amenas, pero me estoy arrepintiendo. Me arrepiento porque dedico muchas horas de preparación y no acabo de ver para qué, ya que ahora los estudiantes no toman ni apuntes, se lo damos todo hecho y al final acabamos trabajando más nosotros y entiendo que el universitario debe de realizar sobre todo un trabajo personal. Ahora imprimen lo que meto en la plataforma y ya tienen los apuntes. Me estoy planteando volver al antiguo método de enseñanza. Porque los alumnos en el mejor de los casos se dedican a copiar alguna cosa sobre el material que han impreso; pero no leen, no buscan, no hacen nada por su cuenta, resulta de lo menos creativo y personal (Sancho y Martínez, 2013, pp. 353-354). 
Una reflexión que conecta con la de un docente de Ciencia Política, que también considera que la facilidad de acceso a la información la hacen de alguna manera menos valiosa y relevante,

En este momento, se puede plantear un profesor decir: -"El gobierno aprueba determinada modificación del código penal" y explicar la ley, y no levantar nadie la mano diciendo: -"Pero oiga ¿no le parece a usted que quizás esta modificación no era necesaria?”. En este sentido veo a los alumnos más pasivos y, por desgracia, en el campo de las Ciencias Sociales se han acostumbrado demasiado a estudiar con apuntes, con fotocopias, a no leer libros [...]. Esto me preocupa, hay que usar Internet y las nuevas tecnologías, etc., pero hay instrumentos clásicos que no se pueden abandonar, y se están perdiendo (Ornellas y Sancho, 2013, p. 39).

A la vez que conlleva la necesidad y transformaciones para el profesorado e importantes desafíos para la institución, como argumenta una profesora de Biología,

ahora lo difícil es saber limitar el conocimiento, la información que hay que dar a los alumnos, saber lo que es fundamental para la formación que se les quiere dar. Esto es algo que al profesorado que está enseñando desde hace muchos años le costará cambiar; hacer este cambio desde querer explicarlo todo a decir "bueno, esto es lo que tenemos que hacer, y lo otro ya lo aprenderán cuando lo necesiten". Yo creo que esto es un cambio de mentalidad importante. El cambio de mentalidad del profesorado tiene que ser importante. Y esto lo veo bastante difícil. Y tal vez eso que decía de que la universidad tiene que ser una institución que tenga un peso social ya no sea así ahora. La universidad tiene que ser un lugar donde se formen unos profesionales que sean competentes, y lo del nivel cultural, eso hay que dejarlo para otro... O a lo mejor tiene que desaparecer, no lo sé (Sánchez de Serdio, 2013, p. 323).

Unos desafíos que, en estos años, han seguido creciendo, de la mano del crecimiento imparable de las tecnologías digitales y el impacto de las políticas neoconservadoras en la universidad. En el próximo y último apartado situamos algunos de ellos.

\section{LA UNIVERSIDAD HACIA EL 2030: APERTURA PARA LA SOSTENIBILIDAD}

La universidad española, como la de otros países, se encuentra en un proceso de reflexión sobre el papel que debe desempeñar para dar respuesta, entre otros, a los retos globales que plantea la Agenda 2030 de las Naciones Unidas. También está sobre la mesa el tema de cómo integrar los Objetivos de Desarrollo Sostenible (ODS) en los distintos ámbitos de acción de la universidad: la formación, la investigación y la extensión universitaria. La agenda incorpora entre sus metas asegurar el acceso igualitario a la educación superior de calidad, incluida la enseñanza universitaria, y 
destaca el papel catalizador de la tecnología y las TIC como uno de los motores en la consecución de los ODS. Específicamente en relación al ODS 4-Educación de calidad se señala que

Es preciso aprovechar las tecnologías de la información y la comunicación (TIC) para reforzar los sistemas educativos, la difusión de conocimientos, el acceso a la información, el aprendizaje efectivo y de calidad, y una prestación más eficaz de servicios. (UNESCO, 2015b, p. 8).

La agenda también es explícita en cuanto al potencial de las TIC en la mejora de las oportunidades de acceso a los niveles superiores de educación,

Un sistema de educación terciaria bien establecido y debidamente regulado, que aproveche la tecnología, los recursos educativos de libre acceso y la educación a distancia, puede mejorar el acceso, la equidad, la calidad y la pertinencia, y también reducir las disparidades entre lo que se enseña en los establecimientos de educación terciaria, incluidas las universidades, y las exigencias de las economías y las sociedades (p. 41).

Una de las estrategias de acción para lograr esta meta pasa por

Elaborar políticas y programas para el suministro de una educación terciaria a distancia de calidad que cuente con financiación suficiente y aproveche la tecnología, incluido Internet, los cursos abiertos en línea de amplia participación y otras modalidades que cumplan las normas reconocidas de calidad, a fin de mejorar el acceso (p. 43).

El informe Opening Up Education: A Support Frameworkfor Higher Education Institutions (Inamorato dos Santos, Punie y Castaño-Muñoz, 2016), propone un marco de referencia encaminado a lograr unos entornos de aprendizaje más abiertos, que proporcionen una enseñanza universitaria de mayor calidad mediante el uso de las TIC y los recursos educativos abiertos. El marco identifica diez dimensiones para una educación superior abierta que pretende servir como una herramienta para ser utilizada por las universidades, los responsables de las políticas educativas y también por otros niveles educativos. El concepto de educación abierta utilizado en el informe va más allá del mero acceso a recursos y materiales educativos disponibles en abierto como pueden ser los OER o MOOCs y hace referencia a

a way of carrying out education, often using digital technologies. Its aim is to widen access and participation to everyone by removing barriers and making learning accessible, abundant, and customisable for all. It offers multiple ways of teaching and learning, building and sharing knowledge. It also provides a variety of access routes 
to formal and non-formal education, and connects the two (Inamorato dos Santos, Punie and Castaño-Muñoz, 2016, p. 12).

En este sentido, una educación abierta permite que todos y cada uno de los individuos, en las diferentes etapas de sus vidas y de desarrollo profesional, puedan tener acceso a oportunidades educativas apropiadas y significativas para ellos. Esto incluye el acceso a contenidos, cursos, orientación, evaluación y certificación, eliminando o disminuyendo significativamente las barreras de acceso y el coste, en consonancia con lo que propone la Agenda 2030. Aunque no podemos perder de vista la necesidad de contar con un mínimo capital cultural y unas condiciones de vida que proporcionen el tiempo y la disposición cognitiva e emocional para poder sacar partido a este tipo de recursos educativos y formativos (Lee y Chen, 2017).

Las diez dimensiones para una educación superior abierta que propone el informe se clasifican en dos categorías: centrales y transversales. Las dimensiones centrales o core dimensions son seis: acceso, contenido, pedagogía, reconocimiento, colaboración e investigación. Las cuatro dimensiones transversales hacen referencia a estrategia, tecnología, calidad y liderazgo. Todos estos aspectos están interrelacionados siendo las dimensiones centrales las que representan el 'qué' de la educación abierta y las transversales las que indican el 'cómo' lograrlo, proporcionando la estructura para la ejecución de las dimensiones centrales. Asimismo, la apertura de la educación será más significativa y podrá realmente promover la reconfiguración de los escenarios de la educación superior si se adopta una visión holística que abarque las diez dimensiones y no se centran los esfuerzos en una única dimensión.

La lógica de la apertura de la educación superior va más allá de la oferta de formación no presencial por universidades tradicionales o abiertas o de impregnar el currículo con TIC. Pasa por dar respuestas a los efectos que el fenómeno de la globalización, una realidad clave en el siglo XXI, ha producido durante las tres últimas décadas en la educación universitaria: la masificación y la necesidad de mejora de las infraestructuras de las instituciones; la extensión del principio del aprendizaje a lo largo lo ancho de la vida para todos (Banks, Au, Ball, Bell et al., 2007); la constitución de un marco europeo de educación superior y la aparición de una red internacional de conocimiento; la predominancia del inglés como el idioma vehicular para la academia y la ciencia; el aumento de las desigualdades entre los sistemas de educación superior nacionales y entre países; la expansión de las TIC o la necesidad del dominio de nuevas competencias por parte de los docentes y los estudiantes, son algunos ejemplos de estos efectos. Existe numerosa bibliografía dedicada al análisis de la naturaleza y las consecuencias de este impacto (Altbach, 2006; Dutton, 2002; King, 2004).

Una educación superior abierta y flexible requiere también conocer las posibilidades de las TIC para mejorar los sistemas de formación y adecuarlos a la sociedad digital en la que vivimos, potenciando cambios estructurales, reduciendo costes y aumentando la flexibilidad en lo que respecta al tiempo y al espacio. A ese 
respecto, el informe NMC Horizon Report: 2017 Higher Education Edition (Adams Becker, Cummins, Davis, Freeman et al., 2017), en la línea de lo señalado en el apartado anterior, apunta el replanteamiento del rol del educador como uno de los desafíos más difíciles. Uno de los retos que resulta definir y mucho más solucionar y que impiden o dificultan la adopción de tecnologías digitales en la enseñanza superior. El informe también destaca los aspectos críticos a tener en cuenta a la hora de crear estrategias efectivas para la promoción de prácticas y tecnologías innovadoras en la enseñanza universitaria en todo el mundo. Según estos autores, algunos de estos aspectos son:

- Las instituciones tienen la necesidad de promover el intercambio de nuevas ideas, identificar modelos de éxito, premiar la innovación didáctica y situar el éxito de los estudiantes en el centro de sus intereses.

- Las instituciones tienen la responsabilidad de ofrecer experiencias de aprendizaje más auténticas, profundas y activas y una formación basada en el desarrollo de habilidades del mundo real para reforzar la empleabilidad.

- La colaboración es la clave para encontrar soluciones efectivas graduales. Las comunidades de prácticas, los grupos de liderazgo multidisciplinares, las redes sociales abiertas pueden ayudar a difundir los enfoques basados en la evidencia.

- A pesar de la proliferación de la tecnología y los recursos digitales, el acceso sigue siendo desigual. Se mantienen, en todo el mundo, las lagunas que están obstaculizando la finalización de los estudios universitarios de los grupos de estudiantes según su estatus socioeconómico, cultura, etnia y género.

- Se necesitan procesos para evaluar la adquisición de habilidades profesionales, competencias, creatividad y pensamiento crítico.

- La fluidez en ámbito digital va más allá de entender cómo usar los recursos tecnológicos. La formación no puede quedarse en la adquisición de habilidades tecnológicas aisladas, sino generar una comprensión profunda y crítica de los entornos digitales.

- El aprendizaje permanente es el alma de la educación superior. Las instituciones se han de plantear priorizar y reconocer el aprendizaje continuo -tanto formal como informal- para sus docentes, personal y estudiantes (p. 2).

Es evidente, por tanto, que el papel de la educación superior resulta clave en la construcción de una sociedad moderna sostenible, pero este papel agrega una complejidad considerable y muchos nuevos desafíos que pasan por comprender el rol más amplio de la educación superior en un mundo globalizado y cómo afecta a la profesión docente y la experiencia de aprendizaje de los estudiantes a lo largo y ancho de la vida. 


\section{CONCLUSIONES}

La sociedad actual como un todo, y la universidad en particular, están experimentando profundas y aceleradas transformaciones que afectan la vida y el trabajo de las personas y el funcionamiento de las instituciones. Una de las fuerzas fundamentales de estos cambios son las tecnologías digitales, cuyo desarrollo y sentido no pueden desvincularse del auge del capitalismo especulativo impulsado por los discursos, políticas y prácticas neoconservadores. Porque, aunque a veces nos parezca lo contrario, como cualquier otra tecnología, no son ellas las que deciden el sentido de su desarrollo y de su uso. Ya que, como argumenta Feenberg (1991, p. 14), la tecnología, cualquier tecnología, no es un destino, ni un imperativo externo a la voluntad de los propios seres humanos, sino una escena de lucha "un parlamento de las cosas en el que formas de civilización alternativas se debaten y deciden".

$\mathrm{Y}$ en estos momentos, como viene siendo habitual, no todo el mundo está representado en ese "parlamento de las cosas", ni por supuesto, tiene el mismo poder de decisión. Como hemos argumentado en la primera parte del artículo, el poder está en manos de las fuerzas neoliberales y economicistas, que imprimen su sello y sus valores también en la educación superior y determinan desde las condiciones de trabajo al valor del conocimiento y la formación. Por su parte, la extensión y generalización de las tecnologías digitales en todos los ámbitos de la vida, como hemos discutido en la segunda parte, están afectando todas las dimensiones del trabajo de los académicos y planteando ingentes desafíos para la universidad. Son estos desafíos a los que nos hemos referido en la tercera parte.

En el panorama que presenta este artículo aparecen un conjunto de problemas y oportunidades. El ingente potencial académico y social de las tecnologías digitales para la mejora de la educación puede verse ensombrecido por su impacto, a veces negativo, tanto en la forma de aprender de los individuos (Carr, 2011) como en su manera de posicionarse frente al mundo (Twenge, 2017). Mientras que las ventajas del acceso que facilitan a la información, la educación y la formación, pueden verse limitadas por la privatización que dificulta su acceso a una parte importante de la población y la falta de oportunidades en el campo laboral.

En definitiva, hemos querido contribuir a dibujar un conjunto complejo de luces y sombras, que nos permitan comprender mejor el mundo en que vivimos para poder, en la medida de nuestras posibilidades, orientarlo hacia el reequilibrio de la desigualdad y la asunción de responsabilices en la defensa de aquello que nos es común (Garcés, 2013). Dado que, para nosotras, el tesoro más preciado de la educación, fundamental y superior, sigue siendo su capacidad para propiciar una vida un poco más digna para toda la humanidad. 


\section{AGRADECIMIENTOS}

ESBRINA- Subjetividades, visualidades y Entornos Educativos Contemporáneos - 2014SGR 0632: http://esbrina.eu

REUNI+D - Red Universitaria de Investigación e Innovación Educativa. Cambios sociales y retos para la educación. MINECO. EDU2015-68718-REDT: http://reunid. $\mathrm{eu}$

\section{FINANCIACIÓN}

Ministerio de Economía, Industria y Competitividad. SEJ2006-01876.

\section{NOTAS}

1. https://es.wikipedia.org/wiki/Anexo:Universidades de Espa\%C3\%B1a

\section{REFERENCIAS BIBLIOGRÁFICAS}

Adams Becker, S., Cummins, M., Davis, A., Freeman, A., Hall Giesinger, C., y Ananthanarayanan, V. (2017). NMC Horizon Report: 2017 Higher Education Edition. Austin, Texas: The New Media Consortium.

Akbaba, Y., y Jeffrey, B. (Ed.) (2017). The Implications of 'New Populism' For Education. London: E \& E Publishing.

Altbach, P. G. (2006). Globalization and the university: Realities in an unequal world. In J. J. F. Forest y P. G. Altbach (Eds.), International Handbook of Higher Education, 1. (121-140). Dordrecht, The Netherlands, Springer.

Ametller, P. (2016). El gran negocio de la privatización de la universidad. Izquierda Diario. Recuperado de https://www. izquierdadiario.es/El-gran-negocio-dela-privatizacion-de-la-universidad.

Anderson, T. (2004). Teaching in an online learning context. En T. Anderson y F. Elloumi (Eds.). Theory and Practice of Online Learning (273-294). Athabasca: Athabasca University.
Banks, J. A., Au, K. H., Ball, A. F., Bell, P., Gordon, E. W., Gutiérrez, K., Heath, S.B., Lee, C. D., Lee, Y., Mahiri, J., Nasir, N.S., Valdes, G. I., y Zhou, M. (2007). Learning in and out of school in diverse environments. life-long, life-wide, lifedeep. The LIFE Center for Multicultural Education. Recuperado de http://life-slc. org/docs/Banks etal-LIFE-DiversityReport.pdf

Carr, N. (2011). The Shallows: What the Internet Is Doing to Our Brains. New York/London: W. W. Norton \& Company.

Chatterton, T., y Newmarch, G. (2017). The future is already here-it's just not very evenly distributed. Interactions, 24(2),4245. doi: https://doi.org/10.1145/3041215

Creus, A., y Gordaliza, M. (2013). Aprender de los cambios: 25 años de ilusión y optimismo crítico. En J. M. Sancho (Coord.), Trayectorias docentes $e$ investigadoras en la universidad. 24 historias de vida profesional. (325337). Barcelona: Dipòsit digital de la Universitat de Barcelona. Recuperado 
de http://diposit.ub.edu/dspace/ handle/2445/44965

Dutton, W. H., y Loader, B. D. (Eds.) (2002). Digital Academe. The New Media and Institutions of Higher Education and Learning. London: Routledge.

Ferreiro, X. (2010). Mercantilización y precarización del conocimiento: el proceso de Bolonia. En Edu-Factory y Universidad Nómada (Comps.), La Universidad en conflicto. Capturas y fugas en el mercado global del siglo del saber (113-142). Madrid: Traficantes de Sueños.

Galcerán, M. (2010). La educación universitaria en el centro del conflicto. En Edu-Factory y Universidad Nómada (comps.), La Universidad en conflicto. Capturas y fugas en el mercado global del siglo del saber (13-39). Madrid: Traficantes de Sueños.

Garcés, M. (2013). El compromís. Barcelona: CCCB.

Goodson, I. F., y Hargreaves, A. (Eds.) (1996). Teachers' professional lives. London: The Falmer Press.

Inamorato dos Santos, A., Punie, Y., y Castaño-Muñoz, J. (2016). Opening Up Education: A Support Framework for Higher Education Institutions. JRC Science for Policy Report, EUR 27938 $E N$. doi: 10.2791/293408.

ITPI (2016). How Privatization Increases Inequality. Recuperado de https:// www.inthepublicinterest.org/wpcontent/uploads/InthePublicInterest InequalityReport Sept2016.pdf

Kenney, M. (2017). Explaining the Growth and Globalization of Silicon Valley: The Past and Today. Recuperado de http:/ www.brie.berkeley.edu/wp-content uploads/2015/01/BRIE-Workingpaper-2017-1.pdf

King, R. (2004). The University in the Global Age. Basingstoke, Palgrave: Macmillan.

Knapp, J. C., y Siegel, D. J. (Ed.) (2009). The business of higher education. Santa Barbara, Calif.: Praeger/ABC-CLIO.
LaFrance, A. (2016). Technology, the Faux Equalizer. Silicon Valley's sunny outlook on technology and opportunity ignores systematic inequalities. Recuperado de https://www.theatlantic.com technologv/archive/2016/03/half-fulltech/476025

Lee, K. S., y Chen, W. (2017). A long shadow: Cultural capital, techno-capital and networking skills of college students. Computers in Human Behavior, 70, 6773.

Martínez, J. S. (2013). Estructura social y desigualdad en España. Madrid: Libros de la Catarata.

Miñana, C., y Rodríguez, J.G. (2003). La educación en el contexto neoliberal. En D. I. Restrepo Botero (Ed.), La falacia neoliberal. Crítica y alternativas (285321). Bogotá: Universidad Nacional de Colombia.

Moulier Boutang, Y. (2012). Cognitive Capitalism. Cambridge: Polity Press.

Ornellas, A., y Sancho, J. M. (2013). Un periplo entre la docencia, la ciencia y la política. En J. M. Sancho (Coord.), Trayectorias docentes e investigadoras en la universidad. 24 historias de vida profesional. (31-42). Barcelona: Dipòsit digital de la Universitat de Barcelona. Recuperado de http://diposit.ub.edu dspace/handle/2445/44965

Pareto, W. (1966). Sociological Writings. London: Pall Mall Press.

Perdomo Reyes, I. (2006). Universidad, ciencia y conocimiento. Prospectiva sobre las consecuencias del Espacio Europeo de Educación Superior. En M. A. Murga y M. P. Quicios (Coord.), La reforma de la Universidad. Cambios exigidos por la nueva Europa (5-27). Madrid: Dykinson.

Rindermann, H. (2018). Cognitive Capitalism: Human Capital and the Wellbeing of Nations. Cambridge: Cambridge University Press.

Rizvi, F. (2016). Privatization in Education: Trends and Consequences. Education 
Research and Foresight Series, 18. Paris: UNESCO. Recuperado de https:// en.unesco.org/node/262287

Rubio, L., Sancho, J. M., y Costas, X. H. (2013). Creatividad, libertad, lengua, política y sociedad. En J. M. Sancho (Coord.), Trayectorias docentes e investigadoras en la universidad. 24 historias de vida profesional. (83-105). Barcelona: Dipòsit digital de la Universitat de Barcelona. Recuperado de http://diposit.ub.edu/ dspace/handle/2445/44965

Romero, F., y Rifà, M. (2013). Análisis crítico de la gestión de procesos en el sistema universitario a través del relato de un docente. En J. M. Sancho (Coord.), Trayectorias docentes e investigadoras en la universidad. 24 historias de vida profesional. (254-267). Barcelona: Dipòsit digital de la Universitat de Barcelona. Recuperado de http://diposit. ub.edu/dspace/handle/2445/44965

Sánchez de Serdio, A. (2013). Del 'referente cultural' al 'profesional unidimensional': treinta años de experiencia. En J. M. Sancho (Coord.), Trayectorias docentes $e$ investigadoras en la universidad. 24 historias de vida profesional. (315324). Barcelona: Dipòsit digital de la Universitat de Barcelona. Recuperado de http://diposit.ub.edu/dspace/ handle/2445/44965

Sancho, J. M. (Coord.) (2013a). Trayectorias docentes e investigadoras en la universidad. 24 historias de vida profesional. Barcelona: Dipòsit digital de la Universitat de Barcelona. Recuperado de http://diposit.ub.edu/dspace/ handle/2445/44965

Sancho, J. M. (2013b). "El que quiere trabajar, y lo quiere hacer honestamente y con dedicación, va a tener su oportunidad”. En J. M. Sancho (Coord.), Trayectorias docentes e investigadoras en la universidad. 24 historias de vida profesional. (106-125). Barcelona: Dipòsit digital de la Universitat de Barcelona.
Recuperado de http://diposit.ub.edu/ dspace/handle/2445/44965

Sancho, J. M., y Hernández, F. (2018). La profesión docente en la era del exceso de información y la falta de sentido. RED. Revista de Educación a Distancia, 56. doi: http://dx.doi.org/10.6018/red/56/4. http://www.um.es/ead/red/56/sancho hernandez.pdf

Sancho, J. M., y Martínez, S. (2013). El sentido de lo fortuito. En J. M. Sancho (Coord.), Trayectorias docentes e investigadoras en la universidad. 24 historias de vida profesional. (349-358). Barcelona: Dipòsit digital de la Universitat de Barcelona. Recuperado de http://diposit. ub.edu/dspace/handle/2445/44965

Senge, P. (1990). La quinta disciplina. El arte y la práctica de la organización abierta al aprendizaje. Barcelona: Granica.

Shavit, Y., Arum, R., Gamoran, A., y Menahem, G. (2007). Stratification in higher education: a comparative study. Stanford, Calif.: Stanford University Press.

Stone, B. (2017). The upstarts: how Uber, Airbnb, and the killer companies of the new Silicon Valley are changing the world. New York: Little, Brown and Company.

Twenge J. (2017). IGen: Why Today's Super-Connected Kids Are Growing Up Less Rebellious, More Tolerant, Less Happy--and Completely Unprepared for Adulthood--and What That Means for the Rest of Us. New York: ATRIA Books.

UNESCO (2011). UNESCO ICT Competency Framework for Teachers. Recuperado de http://unesdoc.unesco.org/ images/0021/002134/213475E.pdf

UNESCO (2015a). Replantear la educación. ¿Hacia un bien común mundial? Recuperado de http://unesdoc.unesco. org/images/0023/002326/232697s.pdf

UNESCO (2015b). Educación 2030. Declaración de Incheon y Marco de Acción para la realización del Objetivo 
de Desarrollo Sostenible 4. Recuperado de http://unesdoc.unesco.org images/0024/002456/245656s.pdt

Vercellone. C. (2010). Capitalismo cognitivo y modelos de regulación de la relación salarial. Algunas enseñanzas del movimiento anti-CPE. En Edu-Factory y Universidad Nómada (Comps.), La Universidad en conflicto. Capturas y fugas en el mercado global del siglo del saber (83-90). Madrid: Traficantes de Sueños.

\section{PERFIL ACADÉMICO Y PROFESIONAL DE LAS AUTORAS}

Juana M. Sancho Gil. Catedrática de Tecnologías Educativas del Departamento de Didáctica y Organización Educativa de la Universidad de Barcelona. Coordina el grupo de investigación -ESBRINA- Subjetividades, visualidades y Entornos Educativos Contemporáneos - 2014SGR 0632; REUNI+D - Red Universitaria de Investigación e Innovación Educativa. Cambios sociales y retos para la educación. MINECO. EDU2015-68718-REDT: http://reunid.eu. Y es miembro de INDAGA'T - Grupo de innovación docente para favorecer la indagación. GINDOC-UB/087: http://www.ub.edu/indagat

E-mail: imsancho@ub.edu

\section{DIRECCIÓN DE LA AUTORA}

Universitat de Barcelona

Passeig de la Vall d'Hebron 171, Edifici Llevant, despatx 228

o8035 Barcelona

Adriana Ornellas. Doctora en Educación por la Universidad de Barcelona. Profesora de los Estudios de Psicología y Ciencias de la Educación de la Universitat Oberta de Catalunya. Coordinadora del grupo de investigación eTIC (Relaciones entre la educación, la ética y las TIC) de la UOC.

E-mail:aornellas@uoc.edu

\section{DIRECCIÓN DE LA AUTORA}

Universitat Oberta de Catalunya

Rambla del Poblenou, 156

o8018 Barcelona

Judith Arrazola Carballo. Doctora en Educación y Sociedad por la Universidad de Barcelona (UB). Máster en Bioética y Derecho. Máster en Intervención en Dificultades de Aprendizaje. Es miembro del Grupo de Investigación ESBRINA Subjetividades, visualidades y entornos educativos contemporáneos -2014SGR 0632: 
http://esbrina.eu, de REUNI+D - Red Universitaria de Investigación e Innovación Educativa. Cambios sociales y retos para la educación. MINECO. EDU2015-68718REDT: http://reunid.eu, y dede INDAGA'T - Grupo de innovación docente para favorecer la indagación. GINDOC-UB/o87: http://www.ub.edu/indagat/

E-mail: juditharrazola@yahoo.es

\section{DIRECCIÓN DE LA AUTORA}

Universitat de Barcelona

Passeig de la Vall d'Hebron 171, Edifici Llevant, despatx 237

o8035 Barcelona

Fecha de recepción del artículo: 15/12/2017

Fecha de aceptación del artículo: 08/01/2018

\section{Como citar este artículo}

Sancho, J. M., Ornellas, A., y Arrazola, J. (2018). La situación cambiante de universidad en la era digital RIED. Revista Iberoamericana de Educación a Distancia, 21(2), pp. 31-49. doi: http://dx.doi.org/10.5944/ried.21.2.20673 